\title{
Coaxial Power Meter Calibration Using a Waveguide Standard*
}

\author{
Glenn F. Engen** \\ Institute for Basic Standards, National Bureau of Standards, Boulder, Colo.
}

(December 8, 1965)

\begin{abstract}
The techniques associated with the calibration of one terminating type power meter in terms of a second terminating meter are useful both in calibration measurements and in the practical application of such devices. These techniques assume a variety of forms and represent an important segment of the microwave art. However their application to the calibration transfer problem between power meters with different input waveguides has long been inhibited by the requirement for an adaptor and the uncertainty which its losses can introduce into the procedure.

This paper describes a method of extending these existing techniques to this more general problem, in which the adaptor losses are only a second order effect. In addition, it provides limits for the error which is thus introduced.
\end{abstract}

Key Words: Power measurement, power calibration, adaptor efficiency.

\section{Introduction}

A large percentage of the power meters used at ultra-high and microwave frequencies are of the terminating type. This means that they (ideally) terminate the waveguide by its characteristic impedance and indicate the power which they absorb.

Given the problem of determining how much power is being delivered by a signal source to a particular load, it is common practice to substitute the terminating power meter for this load and thus measure the power it absorbs. Under ideal conditions this is also the power delivered to the load.

In practice, however, the impedance of the load and power meter will not be equal, and the ratio of the power delivered to the load and to the power meter will differ from unity. The determination of this ratio is of obvious importance in the practical application of terminating power meters. If the object of the measurement is that of calibrating one power meter in terms of another, this ratio determination is often called a "power calibration transfer." Today a variety of techniques are available for dealing with this problem. ${ }^{1}$

In practice, however, most if not all of these methods are limited to the case where the input waveguides to the two terminations (meter and load, etc.,) are of the same type or cross section. The more general

\footnotetext{
*A preliminary account of this work was given at the 1964 Conference on Precision Electromention, March 1965 (New York), and appears in Part 11 of the 1965 IEEE Convention Record (pp. 99-101). Because a different set of boundary conditions were employed, the error limits quoted in Because a different set of boundary conditions were employed, the
the Convention Record differ somewhat from those to be given here.

the Convention Record differ somewhat from those to be given here.

**Radio Standards Engineering Division, National Bureau of Standards, Boulder, Colo.

' For a brief survey of the existing techniques see the author's paper, A variable impedance power meter and adjustable reflection coefficient standard, J. Res. NBS 68C (Engr. and Instr.), No. 1, 7-24 (Jan.-Mar 1964).
}

problem of transferring power calibrations between power meters of rectangular waveguide and coaxial line inputs, for example, has received little attention. The reason for this lies in the implicit requirement for an adaptor and a detailed knowledge of its losses or other characteristics.

This paper will describe a method of effecting such a comparison in which the adaptor losses are only a second order effect and for which limits of error are given. Aside from the adaptors, the method requires little or no instrumentation beyond what is required to compare power meters having the same type of input. Its complexity may be judged by noting that it requires only the application of existing calibration transfer techniques. It does, however, call for two separate measurements which are then averaged to yield the final result. The procedure should prove a useful addition to existing measurement techniques.

\section{General Description}

As a specific example, the calibration of a coaxial bolometer mount in terms of a waveguide "standard" will be considered. The procedure for applying the method to similar problems will then be obvious.

The components to be considered explicitly include the "standard" or calibrated waveguide bolometer mount, the coaxial bolometer mount, and a waveguide to coaxial line adaptor of arbitrary characteristics.

A terminating type power meter may be calibrated either in terms of the "incident" power (power associated with the incident wave in a lossless line) or in terms of the net power absorbed (difference between "incident" and "reflected" powers). Although certain practical arguments can be given in favor of the "incident" power, the net power definitions are based 
on a more fundamental concept. In the case of a bolometer mount the parameter of interest is the effective efficiency, which by definition is the ratio of the bolometrically indicated value to the net power absorbed by the mount. The problem is thus one of measuring the efficiency ${ }^{2}$ of the coaxial mount given the efficiency of the waveguide standard bolometer mount.

As already noted (see footnote 1), techniques exist for effecting such comparisons when the input terminals are alike. More specifically, the comparison procedure yields the ratio of powers actually delivered to the two mounts. This ratio is then multiplied by the power ratio which is observed by the bolometric technique and the ratio of mount efficiencies obtained. Finally if one of these efficiencies is known, the other may be determined.

The calibration procedure, which is the subject of this paper, requires two measurements $m_{1}, m_{2}$, of the efficiency ratios of the adaptor-bolometer mount combinations shown in figures 1 and 2. The actual

2 The term "efficiency" as employed in this paper is to be interpreted in a broad sense and may represent either "effective efficiency" of a bolometer mount, or adaptor "effi ciency" (ratio of net power output to net power input).

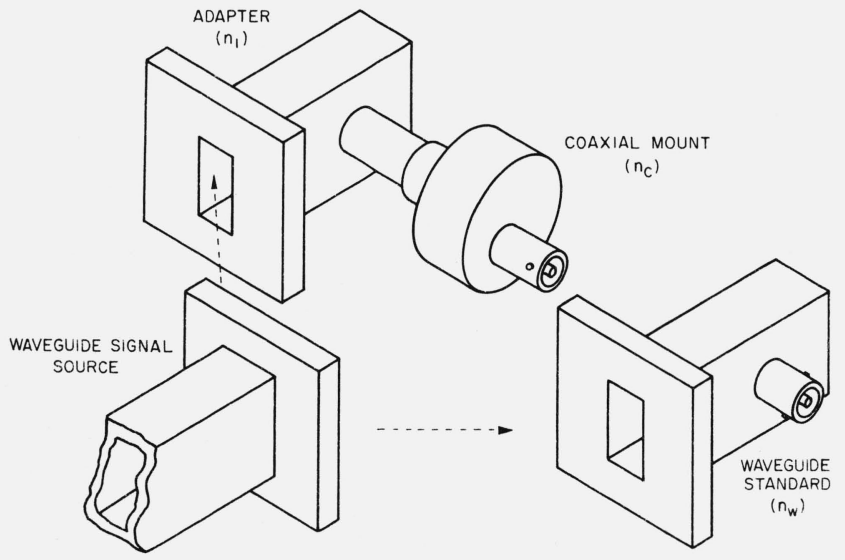

FigURE 1. Illustrating first step of measurement procedure.

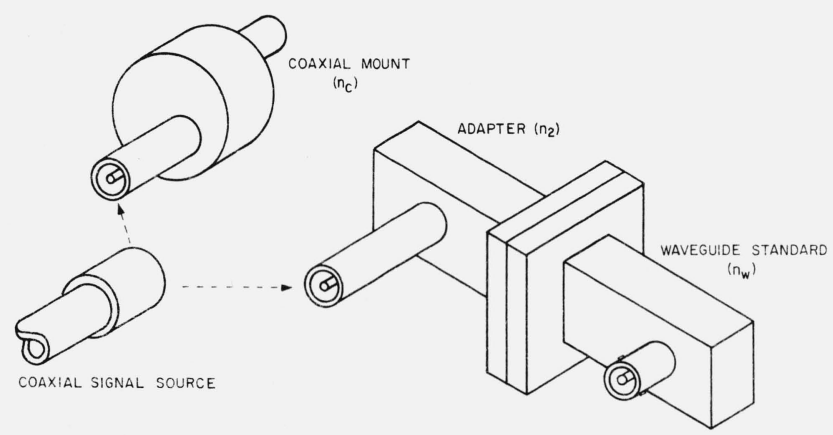

FIGURE 2. Illustrating second step of measurement procedure. measurement procedure is not specified but may consist of any technique, including those listed in reference 1 , which provides these ratios. In figure 1 the adaptor is connected to the coaxial mount such that two similar waveguide terminals are available for the comparison procedure $\left(m_{1}\right)$. In figure 2 the adaptor is connected to the waveguide standard mount, and comparison $\left(m_{2}\right)$ effected at the coaxial terminals. In order to permit these connections both the waveguide and coaxial connectors must be of the "sexless" variety.

If the adaptor were lossless, the efficiency of the adaptor-bolometer mount combinations would equal that of the mounts alone, and either procedure would yield the desired efficiency ratio.

In practice, of course, the adaptor is not lossless. Thus the first measurement, $m_{1}$, yields

$$
m_{1}=\frac{\eta_{1} \eta_{c}}{\eta_{w}},
$$

where $\eta_{c}, \eta_{w}$, and $\eta_{1}$ are respectively the efficiencies of the coaxial mount, standard (waveguide) mount, and adaptor. (Note that the adaptor efficiency is a function of the terminating load impedance and direction of power flow.) Equation (2-1) may be verified by noting that the product found in the numerator is the efficiency of the adaptor-coaxial mount combination. (This is one advantage of basing the efficiency definitions on net power.)

In a similar manner the second measurement, $m_{2}$, yields

$$
m_{2}=\frac{\eta_{c}}{\eta_{2} \eta_{w}},
$$

where $\eta_{2}$ is the adaptor efficiency which obtains during the second measurement. In general $\eta_{1} \neq \eta_{2}$.

It is convenient to regard the determination of the ratio $\eta_{c} / \eta_{w}$ as the object of the measurement procedure. Since, by hypothesis, the efficiency of the standard, $\eta_{w}$, is known, the measurement of this ratio yields the coaxial mount efficiency, $\eta_{c}$.

Inspection of eqs $(2-1)$ and $(2-2)$ shows that the measurement results include the factors $\eta_{1}$ and $\eta_{2}^{-1}$, respectively. Then, because the efficiency cannot exceed unity, $m_{1}$ and $m_{2}$ yield a lower and upper bound to the desired ratio $\eta_{c} / \eta_{w}$.

The geometric mean of $m_{1}$ and $m_{2}$ yields

$$
\sqrt{m_{1} m_{2}}=\frac{\eta_{c}}{\eta_{w}} \sqrt{\frac{\eta_{1}}{\eta_{2}}},
$$

while the quotient

$$
\frac{m_{1}}{m_{2}}=\eta_{1} \eta_{2}=\frac{1}{1+\epsilon} .
$$

Equation (2-4) serves to define the parameter $\epsilon$ implicitly. This parameter is a measure of the adaptor losses and tends to zero as these losses are reduced. 
In summary, the measurement technique consists of making the measurements $m_{1}, m_{2}$, and taking the geometric mean as the desired ratio $\eta_{c} / \eta_{w}$. This includes the approximation $\sqrt{\eta_{1} / \eta_{2}}=1$. A knowledge of either $\eta_{c}$ or $\eta_{w}$ thus permits the determination of the other. As already noted, it is possible to establish limits for the error introduced by this approximation from the fact that the efficiencies cannot exceed unity. Much tighter limits of error, however, can be derived by utilizing the fact that the same adaptor is used in both measurements. More specifically, if reciprocity is satisfied, it is possible to obtain upper and lower limits to this approximation in terms of $\epsilon$ and the impedance conditions which prevail.

\section{Limits of Error}

As noted in the preceding section, the method is based on the approximation $\sqrt{\eta_{1} / \eta_{2}}=1$. The error, thus introduced, depends upon impedance conditions and adaptor losses. Three different modes of operation have been considered and are referred to as Cases I, II, III. For purposes of illustration it is convenient to visualize the procedure in terms of figure 3 , where the adaptor and two bolometer mounts are connected together as shown.

The first measurement, $m_{1}$, consists of separating the assembly at terminal surface $l$ and measuring the ratio of efficiencies of the waveguide mount to the adaptor-coaxial mount assembly. The second measurement, $m_{2}$, is analogous where terminal surface 2 instead of 1 is employed. Let $\Gamma_{w}$ and $\Gamma_{c}$ represent the reflection coefficients of the waveguide and coaxial mounts, and let $\Gamma_{1}$ be the reflection coefficient at terminal surface 1 of the adaptor when terminated by a load $\Gamma_{c}$ (the coaxial mount) as shown in figure 3 . Conversely, let $\Gamma_{2}$ represent the reflection coefficient which obtains at adaptor surface 2 with surface 1 terminated by $\Gamma_{w}$. Finally, let $\left|\Gamma_{a}\right|$ represent the magnitude of the adaptor reflection coefficient. ${ }^{3}$

\footnotetext{
${ }^{3}$ The adaptor reflection coefficient magnitude, $\left|\Gamma_{a}\right|$, is that value which obtains at one side or port of the adaptor with the other end connected to a matched (reflectionless) load. It thus corresponds to the "adaptor VSWR." In general the value of $\left|\Gamma_{a}\right|$ measured at terminal 1 differs from that at terminal 2. For high efficiency adaptors this difference is small and vanishes as the adaptor becomes lossless. Thus for most practical purposes the adaptor may be regarded as characterized by a single value of $\left|\Gamma_{a}\right|$.

The error expressions given for Case $I$ are such as to give the correct limits if the value substituted for $\left|\Gamma_{a}\right|$ is the smaller of the two. Thus, if the larger value is used instead, somewhat wider limits will be obtained.

The failure to identify $\left|\Gamma_{a}\right|$ with either terminal is intentional in that this represents the most general case of practical significance. As will be shown later, tighter limits of error most general case of practical significance. As will be
result if $\left|\Gamma_{a}\right|$ is identified with one terminal or the other.
}

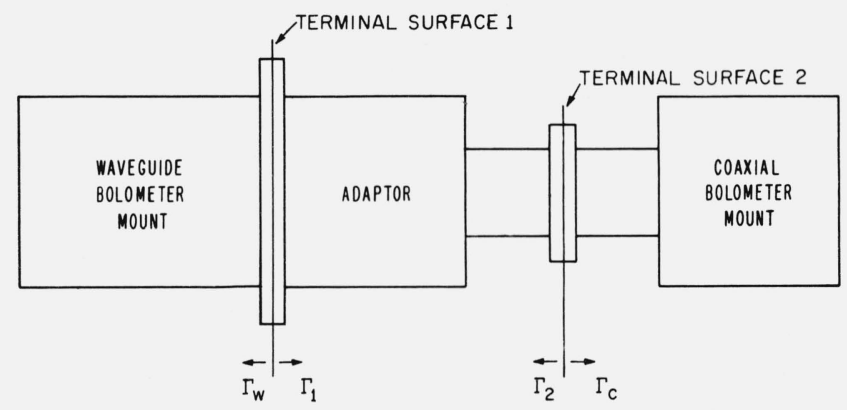

FIGURE 3. Block diagram showing impedance relationships.
The error, $E$, in the different modes of operation is based on the following definition:

$$
E=\sqrt{\frac{\eta_{1}}{\eta_{2}}}-1
$$

Approximate (correct to the order given) limits for $E$ are as follows:

Case I. The impedance conditions are assumed to be completely arbitrary. It will be shown that $E$ lies within the following limits:

$$
\begin{aligned}
& E_{\max }=\frac{\epsilon}{2}\left(\left|\Gamma_{w}\right|+\left|\Gamma_{c}\right|+\left|\Gamma_{a}\right|\right)+\frac{\epsilon^{2}}{8} \\
& E_{\min }=-\frac{\epsilon}{2}\left(\left|\Gamma_{w}\right|+\left|\Gamma_{c}\right|+\left|\Gamma_{a}\right|\right)-\frac{\epsilon^{2}}{8} .
\end{aligned}
$$

Case II. It is assumed that $\Gamma_{2}$ and $\Gamma_{c}$ are equal (in both magnitude and phase) but unknown. This presupposes the incorporation and use of a tuning transformer in one of the components (usually the adaptor) to achieve this condition. It is then possible to express the limits for $E$ in terms of $\left|\Gamma_{1}\right|$ and $\left|\Gamma_{w}\right|$ as follows:

$$
\begin{aligned}
& E_{\max }=\frac{1}{2}\left(\left|\Gamma_{1}\right|+\left|\Gamma_{w}\right|\right)^{2} \quad \text { if }\left|\Gamma_{1}\right|+\left|\Gamma_{w}\right| \leqslant \frac{\epsilon}{2}(3-4) \\
& E_{\max }=\frac{\epsilon}{2}\left(\left|\Gamma_{1}\right|+\left|\Gamma_{w}\right|\right)-\frac{\epsilon^{2}}{8} \quad \text { if }\left|\Gamma_{1}\right|+\left|\Gamma_{w}\right| \geqslant \frac{\epsilon}{2}(3-5) \\
& E_{\min }=-\frac{\epsilon}{2}\left(\left|\Gamma_{1}\right|+\left|\Gamma_{w}\right|\right)-\frac{\epsilon^{2}}{8} .
\end{aligned}
$$

Case III. The reflection coefficients $\Gamma_{1}$ and $\Gamma_{w}$ are assumed to be equal and of known magnitude. The limits for $E$ now become

$$
\begin{gathered}
E_{\max }=2\left|\Gamma_{w}\right|^{2} \quad \text { if }\left|\Gamma_{w}\right| \leqslant \frac{\epsilon}{4} \\
E_{\max }=\epsilon\left|\Gamma_{w}\right|-\frac{\epsilon^{2}}{8} \quad \text { if }\left|\Gamma_{w}\right| \geqslant \frac{\epsilon}{4} \\
E_{\min }=-\epsilon\left|\Gamma_{w}\right|-\frac{\epsilon^{2}}{8} .
\end{gathered}
$$

The error limits for Case III may thus be obtained from Case II by letting $\left|\Gamma_{1}\right|=\left|\Gamma_{w}\right|$.

Although the errors associated with Cases II and III are somewhat smaller than in Case I, a more important argument in their favor is the simplification which they permit in the intercomparison measurements $\left(m_{1}, m_{2}\right)$. Generally speaking, one of these measurements will be simplified if $\Gamma_{2}=\Gamma_{c}$ or if $\Gamma_{1}=\Gamma_{w}$. 
In Case II, for example, the first step is to adjust the adaptor (transformer) such that $\Gamma_{2}=\Gamma_{c}$. This permits a simplification in the measurement $m_{2}$. Although measurement $m_{1}$ must in general account for $\Gamma_{1} \neq \Gamma_{w}$, this problem (along with the measurement of $\left|\Gamma_{1}\right|$ and $\left.\left|\Gamma_{w}\right|\right)$ is more easily handled in waveguide than in coaxial line (at least at higher frequencies!).

In Case III only one reflection coefficient magnitude $\left|\Gamma_{w}\right|\left(=\left|\Gamma_{1}\right|\right)$ enters the error expressions and, all else being equal, gives the smallest error. The problem of making the calibration transfer between unequal impedances $\left(m_{2}\right)$, however, has been shifted to the coaxial side where it is usually less convenient. An important application of Case III will be discussed in the following section.

\section{Extension to Type $\mathbf{N}$ Connectors}

The foregoing techniques are based on the requirement that the connectors satisfy the "sexless" condition. Although a number of precision coaxial connectors are now available which meet this criterion, the extensively employed Type $\mathrm{N}$ does not. The technique may be extended to cover this case as follows.

It is now convenient to visualize the problem in terms of the assembly shown in figure 4. The first step of the measurement procedure is identical to that already described. However when the structure is separated at terminal surface 2, a problem is encountered in that it is not possible to mate both of these surfaces with a third one.

The solution requires the introduction of additional transitions and calibration transfer measurements as shown in figures $5 \mathrm{a}$ and $5 \mathrm{~b}$. The measurement " $m_{2}$ " is thus replaced by a pair of measurements $\left(m_{2 a}, m_{2 b}\right)$ which, by inspection, yield the ratios: $\eta_{f} \eta_{c} / \eta_{2} \eta_{w}$ and $\eta_{c} / \eta_{m} \eta_{2} \eta_{w}$, where $\eta_{f}$ and $\eta_{m}$ are the efficiencies of the additional transitions (adaptors) as shown. Since these efficiencies cannot exceed unity, it is possible to write

$$
\frac{\eta_{f} \eta_{c}}{\eta_{2} \eta_{w}}=m_{2 a}<m_{2}<m_{2 b}=\frac{\eta_{c}}{\eta_{m} \eta_{2} \eta_{w}} .
$$

The pair of measurements $\left(m_{2 a}, m_{2 b}\right)$ thus yields upper and lower limits to $m_{2}$. If their arithmetic average is used in place of $m_{2}$, equation $(2-3)$ becomes

$$
\sqrt{m_{1} \frac{\left(m_{2 \prime}+m_{2 b}\right)}{2}}=\frac{\eta_{c}}{\eta_{w}} \sqrt{\frac{\eta_{1}}{\eta_{2}}} \sqrt{\frac{1}{2}\left(\eta_{f}+\frac{1}{\eta_{m}}\right)} .
$$

The error introduced by assuming $\sqrt{\eta_{1} / \eta_{2}}=1$ has already been described. It is easily shown that the additional error in assuming $\sqrt{\frac{1}{2}\left(\eta_{f}+1 / \eta_{m}\right)}=1$ is within the approximate limits $\pm_{2}^{1}\left(m_{2 b}-m_{2 a}\right) /\left(m_{2 b}+m_{2 a}\right)$. As an illustration, it will be assumed that $m_{2 a}$ and $m_{2 b}$

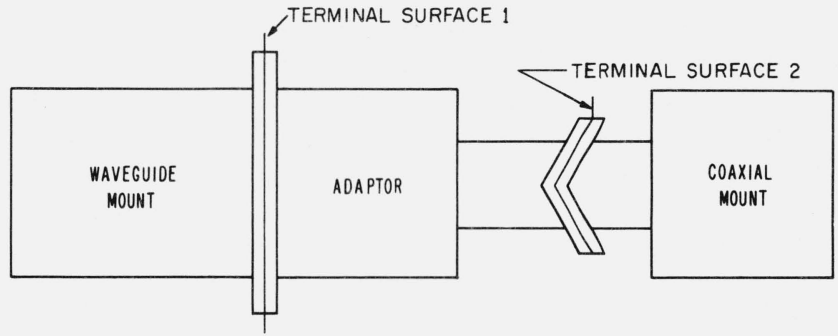

FIGURE 4. Block diagram illustrating problem implicit in type $\mathrm{N}$ connectors.

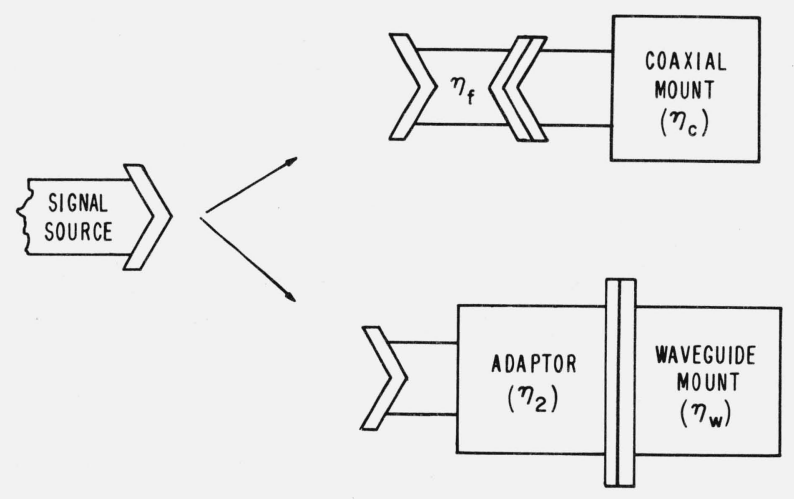

(a)
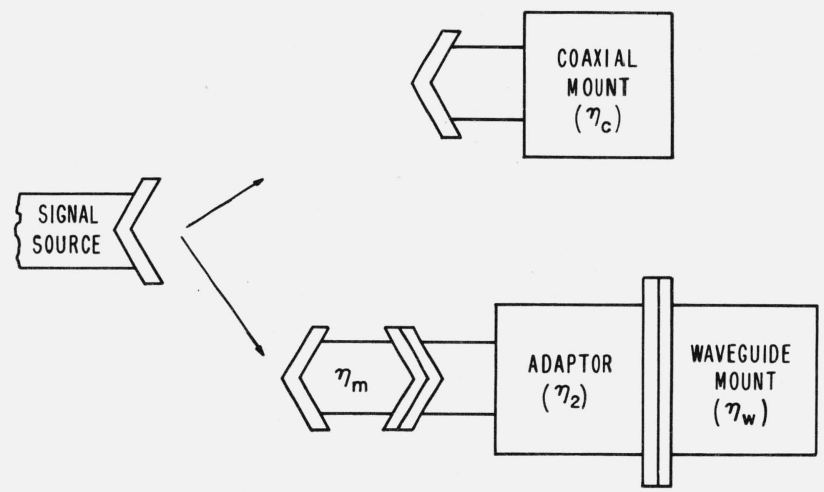

FIGURE 5. Adaptor configuration for measurements $\mathrm{m}_{2 \mathrm{a}}$ and $\mathrm{m}_{2 \mathrm{~b}}$.

differ by 1 percent. The average will then differ from $m_{2}$ by no more than \pm 0.5 percent. Finally, this value is averaged with $m_{1}$. Since $m_{1}$ and $m_{2}$ are nominally equal, a \pm 0.5 percent uncertainty in $m_{2}$ will give a \pm 0.25 percent error in the final result. 


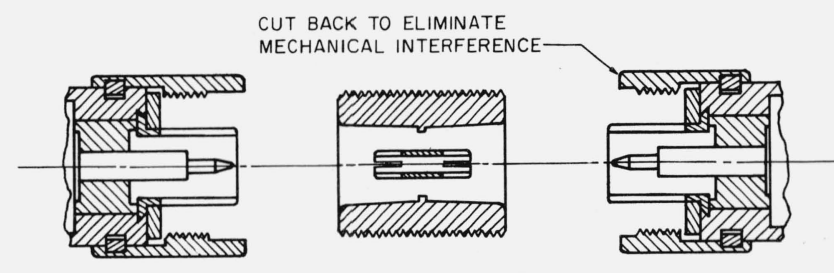

(a)

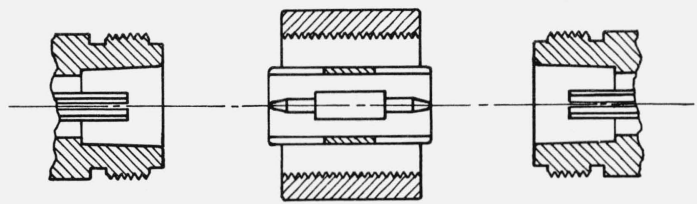

(b)

FIGURE 6. Transition sections required in extending technique to type $\mathrm{N}$ connectors.

Although these additional transitions can be made by commercially available components, it is desirable in practice to keep the associated losses as small as possible. Figures $6 \mathrm{a}$ and $6 \mathrm{~b}$ show adaptors which have been built to satisfy this requirement. In particular it should be noted that the center conductor is supported only at its ends by the mating connectors.

The Type $\mathrm{N}$ connector suffers from a number of limitations including impedance discontinuities, which become increasingly important at high frequencies, and the lack of a well defined terminal plane or surface. As a consequence it is difficult to give a meaningful definition to impedance at the connector interface.

These considerations strongly suggest the use of a power calibration transfer procedure (measurement $m_{2}$ ) which is independent of the connector discontinuity. Such a technique has been described in a previous paper. ${ }^{4}$ The complete procedure thus comes under Case III, where the impedances are matched and measured at the waveguide side (terminal 1), leaving the power calibration transfer between unequal impedances to be effected at terminal 2. (Note that according to the point of view adopted in the preceding paragraph, Case II cannot be applied because the impedance discontinuity makes it impossible to recognize when $\Gamma_{c}=\Gamma_{2}$.)

An alternative approach to using the Type $\mathrm{N}$ connector is based on the choice of reference plane indicated in figure 7. (This convention is called out in MIL Spec. C39012/1-5.)

If this point of view is adopted, it is desirable (in theory) to eliminate the shoulder in the outer conductor transition piece of figure 6 a such that the two outside conductors are in physical contact.

The adaptor is thus, by definition, absorbed by the two Type $\mathrm{N}$ male connectors such that $\eta_{f}$ may be given

${ }^{4} \mathrm{G}$. F. Engen, A transfer instrument for the intercomparison of microwave power meters, IRE Trans. Instr. I-9, No. 2, 202-208 (Sept. 1960).

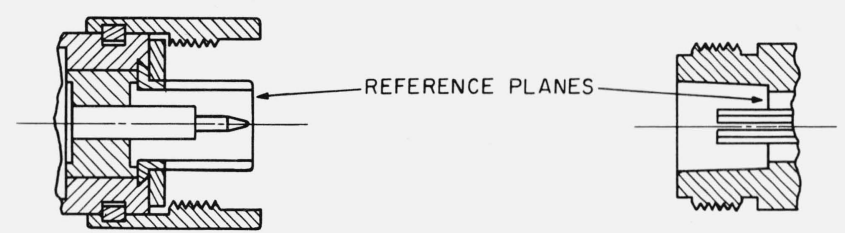

FiguRE 7. Possible reference plane for type $\mathrm{N}$ connector.

the value unity. ${ }^{5}$ The measurement $m_{2 a}$ thus becomes $m_{2}$, and measurement $m_{2 b}$ is no longer required.

It is important to note that, according to this convention, the Type $\mathrm{N}$ male connector may be mated with either male or female, but this is not true of the Type $\mathrm{N}$ female. Thus this technique is limited to the case where the coaxial meter is fitted with the Type $\mathrm{N}$ male connector.

This convention also makes it possible to make the comparison measurements under Case II since the two assemblies shown in figure 5 a may be adjusted (assuming tuning is available) for equal input impedances.

This alternative procedure is somewhat easier to implement, but more restricted in its application and interpretation.

\section{Derivation of Error Expressions}

A microwave measurements problem of long standing finds its solution in the foregoing techniques. However if these procedures are to be accepted by the scientific community, it appears desirable to record the derivation in sufficient detail to demonstrate its validity. Moreover, while the arguments are rather long, they have a potential application to related problems and are thus of some interest in their own right. The object of this section is the derivation of equations (3-2)-(3-9). (The reader who is willing to accept this "on faith" may, without loss of continuity, proceed to the next section.)

A complete description of the adaptor (at one frequency) requires six parameters - the real and imaginary components of its impedance matrix, for example. In addition, the adaptor efficiencies also depend upon the complex impedance of the terminating loads (bolometer mounts). Thus a total of 10 parameters is involved.

Obviously, if these 10 parameters were known, they would permit an exact determination of $E$. In practice, however, many of these parameters, especially those pertaining to the adaptor, do not lend themselves to ready measurement. Indeed, the value of this technique rests in a large measure upon its ability to provide limits for $E$ with a minimum of supplementary information.

\footnotetext{
${ }^{5}$ This ignores any losses introduced by the failure to perfectly mate the outer conductors.
} 


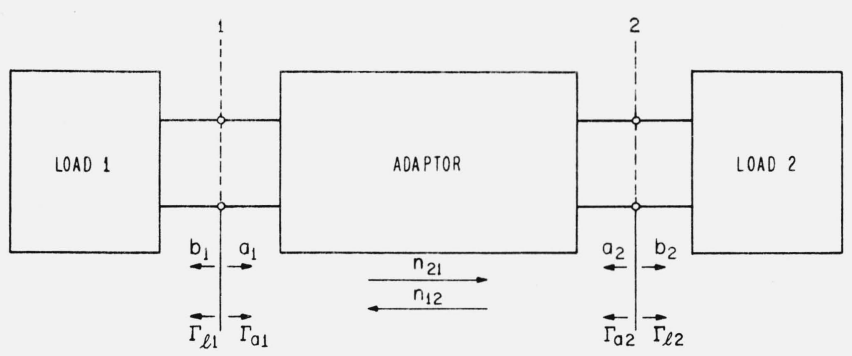

FigURE 8. Block diagram for error evaluation.

Although the derivations are rather involved, it is easy to show that the error, $E$, is of second order. As already noted, the error vanishes in the ideal case of a lossless adaptor. The parameter, $\epsilon$, is one measure or indication of the extent by which the adaptor fails to satisfy this condition. Thus the error tends to zero as $\epsilon$ goes to zero.

On the other hand, if an impedance match $(\Gamma=0)$ is assumed for the different components, $\eta_{1}=\eta_{2}$ and again the error vanishes. This is also an idealization, but one which is approximately satisfied in practice. Since the error vanishes under either of these conditions, the error expressions may be expected to involve the product of $\epsilon$ and the $\Gamma^{\prime}$ 's. ${ }^{6}$

The problem may be formulated in a variety of ways; a convenient one is in terms of the normalized complex incident and emergent "voltage" wave amplitudes, $a_{1}, a_{2}, b_{1}, b_{2}$, as shown in figure 8 and the constants, $\alpha, \beta, \gamma$, of the linear fractional transformation which relate the ratio $b_{1} / a_{1}$ to $a_{2} / b_{2}$. That is,

$$
\frac{b_{1}}{a_{1}}=\frac{\alpha \frac{a_{2}}{b_{2}}+\beta}{\gamma \frac{a_{2}}{b_{2}}+1} .
$$

In terms of the more familiar scattering matrix notation,

$$
\begin{aligned}
\alpha & =S_{12}^{2}-S_{11} S_{22} \\
\beta & =S_{11} \\
\gamma & =-S_{22} \\
\alpha-\beta \gamma & =S_{12}^{2},
\end{aligned}
$$

and

$$
\begin{aligned}
& b_{1}=S_{11} a_{1}+S_{12} a_{2} \\
& b_{22}=S_{12} a_{1}+S_{22} a_{22} .
\end{aligned}
$$

It should be noted that reciprocity is assumed and impedance normalization made such that $S_{12}=S_{21}$. The complex constants $\alpha, \beta, \gamma$ thus provide a complete description of the adaptor.

The condition that the adaptor be source-free or passive imposes certain conditions on the parameters $\alpha, \beta, \gamma$. Under the assumed normalization the scattering matrix $S$ satisfies the matrix equation: ${ }^{7}$

$$
\operatorname{Det}\left(1-S^{*} S\right) \geqslant 0 \text {. }
$$

[In this equation $(*)$ represents the Hermitian conjugate.]

For a two-arm junction this reduces to

$$
\begin{aligned}
& \left(1-\left|S_{11}\right|^{2}-\left|S_{12}\right|^{2}\right)\left(1-\left|S_{12}\right|^{2}-\left|S_{22}\right|^{2}\right) \\
& -\left|S_{11} S_{12} *+S_{12} S_{22} *\right|^{2} \geqslant 0,
\end{aligned}
$$

and in terms of $\alpha, \beta, \gamma$ becomes

$$
1-|\gamma|^{2}-|\beta|^{2}+|\alpha|^{2}-2|\alpha-\beta \gamma| \geqslant 0 .
$$

If eq $(5-6)$ is multiplied by $1-|\gamma|^{2}$, the resulting relation can be expressed in the form

$$
\left(1-|\gamma|^{2}-|\alpha-\beta \gamma|\right)^{2}-\left|\beta-\alpha \gamma^{*}\right|^{2} \geqslant 0 .
$$

It will also be shown in the next section [see eq (5-15)] that $1-|\gamma|^{2}-|\alpha-\beta \gamma| \geqslant 0$. Thus eq (5-7) may be written

$$
1-\frac{|\alpha-\beta \gamma|}{1-|\gamma|^{2}} \geqslant \frac{\left|\beta-\alpha \gamma^{*}\right|}{1-|\gamma|^{2}} \geqslant 0
$$

Finally, it will prove convenient to make the following definitions:

$$
\begin{aligned}
1-\frac{|\alpha-\beta \gamma|}{1-|\gamma|^{2}} & =x, \\
\frac{\beta-\alpha \gamma^{*}}{1-|\gamma|^{2}} & =y .
\end{aligned}
$$

Note that $x$ is real and positive while $y$ is complex. Equation (5-8) thus becomes

$$
x \geqslant|y| \geqslant 0 .
$$

\subsection{Analysis of a Special Case}

Let $\eta_{21}$ and $\eta_{12}$ represent the adaptor efficiencies under the two conditions shown in figure 8 , and let $\Gamma_{l 1}, \Gamma_{a 1}, \Gamma_{a 2}, \Gamma_{l 2}$ represent the reflection coefficients which obtain at the different terminals as indicated. For example, $\Gamma_{l 1}=a_{1} / b_{1}=1 / \Gamma_{a 1}$, etc. (This notation is somewhat more general than that employed in the earlier section and is introduced to simplify the application to other problems.)

${ }^{7}$ C. G. Montgomery, R. H. Dicke, and E. M. Purcell, Principles of Microwave Circuits (McGraw-Hill Book Co., Inc., New York, N.Y., 1948). 
By definition,

$$
\eta_{21}=\frac{\text { Net power delivered to load } 2}{\text { Net power input at terminal } 1}=\frac{\left|b_{2}\right|^{2}-\left|a_{2}\right|^{2}}{\left|a_{1}\right|^{2}-\left|b_{1}\right|^{2}},
$$

and similarly,

$$
\eta_{12}=\frac{\left|b_{1}\right|^{2}-\left.a_{1}\right|^{2}}{\left|a_{2}\right|^{2}-\left|b_{2}\right|^{2}}
$$

The analysis of Case I is facilitated by considering first the special case where $\Gamma_{l 1}=\Gamma_{l 2}=0$. Applying these conditions (in turn) the adaptor efficiencies may be written in terms of $\alpha, \beta, \gamma$ as follows:

$$
\begin{aligned}
& \eta_{21}=\frac{|\alpha-\beta \gamma|}{1-|\beta|^{2}}, \\
& \eta_{12}=\frac{|\alpha-\beta \gamma|}{1-|\gamma|^{2}} .
\end{aligned}
$$

The assertion made in connection with eq $(5-8)$ is now evident, since $\eta_{12} \leqslant 1$.

It will prove instructive to consider the maximum and minimum values of $\eta_{21}$ assuming $\eta_{12}$ and $|\gamma|$ are given. From eqs (5-14) and (5-15),

$$
\gamma_{l 21}=\eta_{21} \frac{1-|\gamma|^{2}}{1-|\beta|^{2}}
$$

By inspection $\eta_{21}$ increases in value when $|\beta|^{2}$ increases and conversely. Although the value of $|\beta|^{2}$ is not given, it is possible to determine limits for its value in terms of $\eta_{12}$ and $|\gamma|$ :

$$
\begin{aligned}
& |\beta|^{2} \equiv \frac{\left|\left(\beta-\alpha \gamma^{*}\right)+(\alpha-\beta \gamma) \gamma^{*}\right|^{2}}{\left(1-|\gamma|^{2}\right)^{2}} \\
& \leqslant\left[\frac{\left|\beta-\alpha \gamma^{*}\right|}{1-|\gamma|^{2}}+\frac{|\alpha-\beta \gamma| \cdot|\gamma|}{1-|\gamma|^{2}}\right]^{2} .
\end{aligned}
$$

thus,

$$
|\beta|^{2} \leqslant\left(|y|+\eta_{12}|\gamma|\right)^{2}
$$

The maximum value of $|\beta|^{2}$ will occur for the maximum value of $y$, that is when $|y|=x=1-\eta_{12}$. Therefore,

$$
|\beta|_{\max }^{2}=\left(1-\eta_{12}+\eta_{12}|\gamma|\right)^{2} .
$$

Substitution in eq (5-16) yields

$$
\eta_{21(\max )}=\frac{1+|\gamma|}{2-\eta_{12}(1-|\gamma|)} .
$$

In order to determine the minimum value of $|\beta|^{2}$, it is convenient to write eq $(5-17)$ in the form

$$
|\beta|^{2}=\left|y+\frac{(\alpha-\beta \gamma) \gamma^{*}}{1-|\gamma|^{2}}\right|^{2} .
$$

Since the only restriction on $y$ is a limit to its magnitude, $|\beta|^{2}$ can be made to vanish by a suitable choice of $y$ for small values of $|\gamma|$. Comparison with eqs (5-8) and (5-15) shows that this is possible provided

$$
|\gamma| \leqslant \frac{1-\eta_{12}}{\eta_{12}}
$$

For larger values of $|\gamma|$, the minimum value of $|\beta|^{2}$ is given by

$$
|\beta|_{\min }^{2}=\left[\eta_{12}|\gamma|-\left(1-\eta_{12}\right)\right]^{2} .
$$

Substitution of these results into eq $(5-16)$ yields

$$
\begin{array}{ll}
\eta_{21(\min )}=\eta_{12}\left(1-|\gamma|^{2}\right) & \text { if }|\gamma| \leqslant \frac{1-\eta_{12}}{\eta_{12}}, \\
\eta_{21(\min )}=\frac{(1-|\gamma|)}{2-\eta_{12}(1+|\gamma|)} & \text { if }|\gamma| \geqslant \frac{1-\eta_{12}}{\eta_{12}} .
\end{array}
$$

Figure 9 shows a plot of eqs (5-20), (5-24), (5-25) with $|\gamma|$ equal to 0.2 .

The area bounded by the $\eta_{21(\max )}, \eta_{21(\min )}$ curves, and the $\eta_{21}$ axis represents possible combinations of $\eta_{21}$ and $\eta_{12}$ for the given choice of $|\gamma|$. In practice neither $\eta_{21}$ nor $\eta_{12}$ is known, but they are connected by the relation

$$
\eta_{21} \eta_{12}=\frac{1}{1+\epsilon} .
$$

This relationship is also plotted in figure 9 where $\epsilon=0.2$. (This value of $\epsilon$ is not representative, but chosen to better exhibit the characteristics of the problem.)

The final object of this section is to determine maximum and minimum values for the ratio $\eta_{21} / \eta_{12}$ in terms of $\epsilon$ and $|\gamma|$. By inspection it is evident that this occurs at the intersection of eq $(5-26)$ with the $\eta_{21(\max )}$ and $\eta_{21(\min )}$ loci.

The maximum value of $\eta_{21} / \eta_{12}$ may be calculated from eqs $(5-20)$ and $(5-26)$ and is given by

$$
\left.\frac{\eta_{21}}{\eta_{12}}\right|_{\max }=1+\frac{\epsilon|\gamma|+\frac{\epsilon^{2}}{4}(1+|\gamma|)^{2}}{1+\epsilon} .
$$

In a similar way,

$$
\left.\frac{\eta_{21}}{\eta_{12}}\right|_{\min }=1-|\gamma|^{2} \quad \text { if }|\gamma| \leqslant \frac{\epsilon}{2+\epsilon} .
$$

$$
\left.\frac{\eta_{21}}{\eta_{12}}\right|_{\min } ^{=1}-\frac{\epsilon|\gamma|-\frac{\epsilon^{2}}{4}(1-|\gamma|)^{2}}{1+\epsilon} \quad \text { if }|\gamma| \geqslant \frac{\epsilon}{2+\epsilon} .
$$




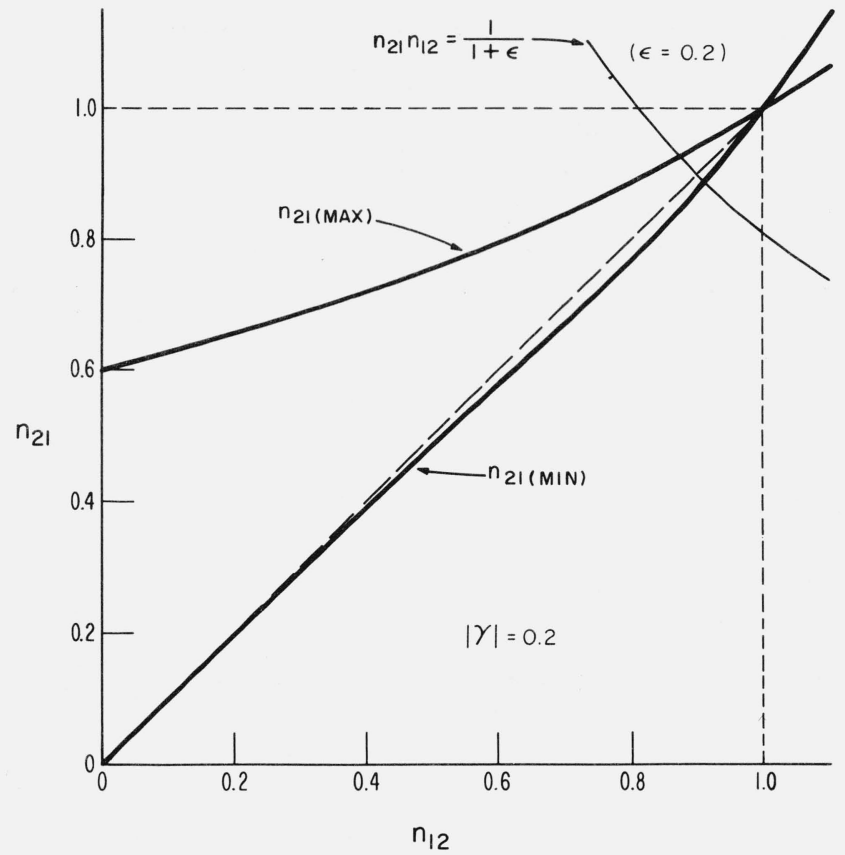

FigURE 9. Plot showing limits for $\eta_{21}$ with $|\gamma|=0.2$.

These last three equations give the limits ${ }^{8}$ for $\eta_{21} / \eta_{12}$ in terms of $\epsilon$ and $|\gamma|$ subject to the condition that both loads (power meters) are matched $\left(\Gamma_{l 1}=\Gamma_{l 2}=0\right)$.

By definition, [eq (5-2)], $|\gamma|$ represents the reflection coefficient magnitude "looking into" adaptor terminal 2 with terminal 1 connected to a matched load. Figure 10 shows the reduction in the spread of possible values for $\eta_{21} / \eta_{12}$ when $\gamma=0$.

\subsection{Case I}

In Case I each of the two loads (power meters) is assumed to have an arbitrary reflection of known magnitude.

The generalization of the foregoing results to Case I is simplified by use of the following artifice:

Returning to figure 8 , it is possible to construct an equivalent circuit of loads 1 and 2 as shown in figure 11 . The characteristics of the "lossless transformers" are adjusted to duplicate the impedances of the respective loads. Substitution into figure 8 results in the

\footnotetext{
${ }^{8}$ It is of interest to compare this result with that which would be obtained if different adaptors were involved in the two measurements (as was done in extending the technique to Type $\mathrm{N}$ connectors). In the latter case the only condition which could be used is that the efficiencies do not exceed unity which leads to

$$
\left.\frac{\eta_{21}}{\eta_{12}}\right|_{\max }=1+\left.\epsilon \cdot \frac{\eta_{21}}{\eta_{12}}\right|_{\min }=\frac{1}{1+\epsilon} .
$$

Although these expressions result in first order errors, they are of some interest in that they are completely independent of the impedance conditions.
}

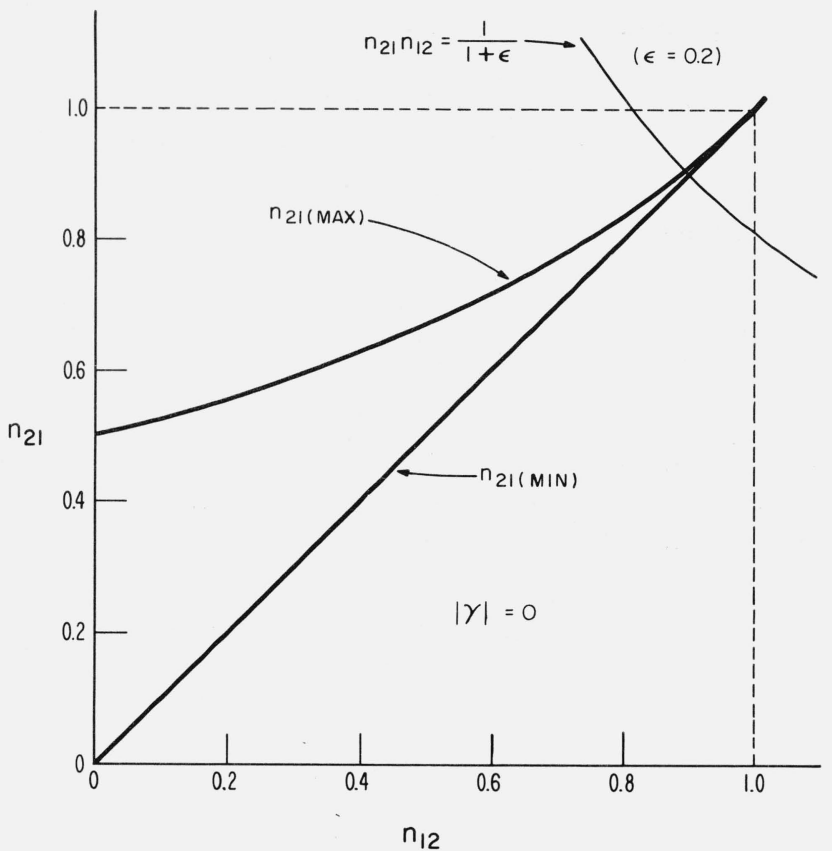

FIGURE 10. Plot showing limits for $\eta_{21}$ with $\gamma=0$.

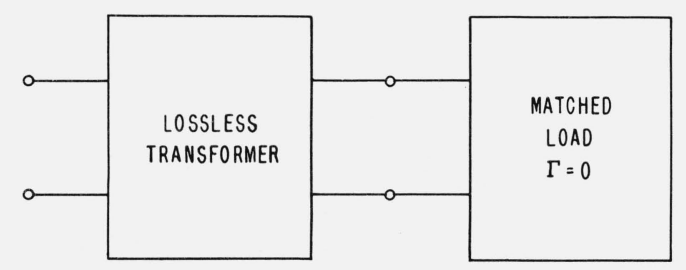

FigURE 11. "Equivalent circuit" of unmatched loads (power meters).

configuration shown in figure 12 . It is now convenient to shift the terminal surfaces from the unprimed to the primed positions such that the lossless transformers become part of the adaptor. Because the efficiencies $\eta_{21}, \eta_{12}$ are based on net power flow, their values are invariant to this shift in terminal surfaces. The results of the preceding section may thus be applied provided an appropriate change is made in $|\gamma|$ to account for the addition of the lossless transformers to the adaptor.

Let $\gamma^{\prime}$ represent the value $\gamma$ takes when the terminal surfaces are shifted to the primed positions, and let $\Gamma_{l 1}, \Gamma_{l 2}$ represent the reflection coefficients of the two loads: By definition $\gamma^{\prime}$ is equal to the ratio $b_{2}^{\prime} / a_{2}^{\prime}$ which obtains at terminal $2^{\prime}$ assuming the matched load is removed and the assembly is excited at this port. The transformer bounded by terminals $\left(2^{\prime}-2\right)$ is thus terminated by a load of reflection coefficient $\Gamma_{a 2}=b_{2} / a_{2}$, while terminal 1 of the adaptor is terminated by a load $\Gamma_{l 1}=a_{1} / b_{1}$. 


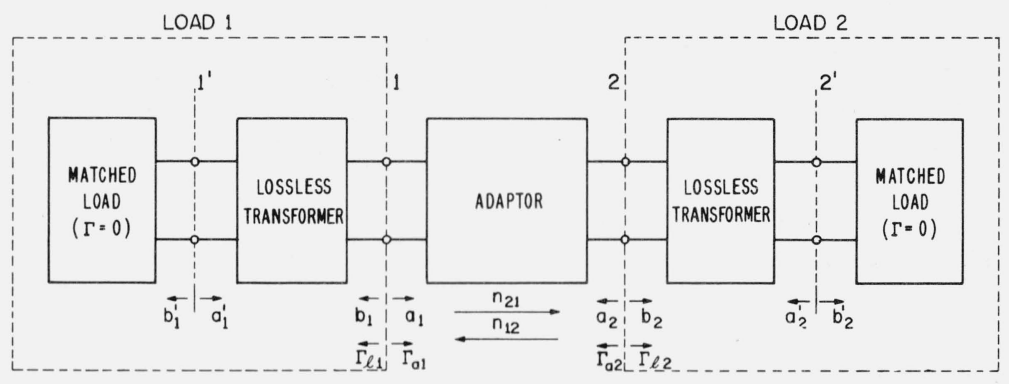

FIGURE 12. Equivalent circuit used in extending analysis to unmatched loads.

From eq (5-1),

$$
\Gamma_{a 2}=\frac{\alpha \Gamma_{l 1}-\gamma}{-\beta \Gamma_{l 1}+1}
$$

By hypothesis, the argument of $\Gamma_{l 1}$ is unknown (as well as the arguments of $\alpha, \beta, \gamma)$. The problem is now one of determining the maximum value of $\left|\Gamma_{a 2}\right|$ as the argument of $\Gamma_{l 1}$ varies. For convenience the adaptor will be assumed lossless. The parameters $\alpha, \beta, \gamma$ then satisfy the conditions

$$
\begin{gathered}
\beta-\alpha \gamma^{*}=0 \\
|\alpha|=1
\end{gathered}
$$

which follow from eq $(5-8)$ when equality is assumed. These conditions may now be substituted back into eq $(5-30)$ and the magnitude, $\left|\Gamma_{a 2}\right|$, differentiated with respect to the argument of $\Gamma_{l 1}$. This leads to ${ }^{9}$

$$
\left|\Gamma_{a 2}\right|_{\max }=\frac{|\gamma|+\left|\Gamma_{l 1}\right|}{1+\left|\gamma \Gamma_{l 1}\right|} .
$$

By a straightforward extension of these arguments $\left|\gamma^{\prime}\right|_{\max }$ is given by

$\left|\gamma^{\prime}\right|_{\max }=\frac{\left|\Gamma_{a 2}\right|_{\max }+\left|\Gamma_{l 2}\right|}{1+\left|\Gamma_{l 2}\right|\left|\Gamma_{a 2}\right|_{\max }}$

$$
=\frac{\left|\Gamma_{l 1}\right|+\left|\Gamma_{l 2}\right|+|\gamma|+\left|\Gamma_{l 1} \Gamma_{l 2} \gamma\right|}{1+\left|\Gamma_{l 1} \Gamma_{l 2}\right|+\left|\gamma \Gamma_{l 1}\right|+\left|\gamma \Gamma_{l 2}\right|}
$$

As previously noted, the adaptor has been assumed lossless for this calculation. Because of losses, the actual value $\left|\gamma^{\prime}\right|_{\max }$ will be somewhat smaller. In most practical applications the difference will be small, and in any case the use of eq (5-33) leads to error expressions which err on the side of being too large.

${ }^{9}$ Alternatively, eq $(5-32)$ may be obtained by noting that $\left|\Gamma_{l 1}\right|=$ constant represents a
circle in the $\Gamma_{l 1}$ plane. The corresponding locus of $\Gamma_{a 2}$ is also a circle whose radius and circle in the $\Gamma_{11}$ plane. The corresponding locus of $\Gamma_{a 2}$ is also a circle whose radius and displacement from the origin may be obtained in terms of $\alpha, \beta, \gamma$, and $\left|\Gamma_{11}\right|$. The maximum value of $\left|\Gamma_{a 2}\right|$ is given by the sum of this radius and displacement.
Equation (5-33) is now substituted into eq (5-27), (5-28), (5-29), the square root taken, and only the lowest order terms retained. This results in the approximate expressions: ${ }^{10}$

$$
\begin{gathered}
\left(\frac{\eta_{21}}{\eta_{12}}\right)_{\max }^{1 / 2}=1+\frac{\epsilon}{2}\left(\left|\Gamma_{l 1}\right|+\left|\Gamma_{l 2}\right|+|\gamma|\right)+\frac{\epsilon^{2}}{8}, \\
\left(\frac{\eta_{21}}{\eta_{12}}\right)_{\text {min }}^{1 / 2}=1-\frac{1}{2}\left(\left|\Gamma_{l 1}\right|+\left|\Gamma_{l 2}\right|+|\gamma|\right)^{2} \\
\quad \text { if }\left|\Gamma_{l 1}\right|+\left|\Gamma_{l 2}\right|+|\gamma| \leqslant \frac{\epsilon}{2}, \\
\left(\frac{\eta_{21}}{\eta_{12}}\right)_{\text {min }}^{1 / 2}=1-\frac{\epsilon}{2}\left(\left|\Gamma_{l 1}\right|+\left|\Gamma_{l 2}\right|+|\gamma|\right)+\frac{\epsilon^{2}}{8} \\
\text { if }\left|\Gamma_{l 1}\right|+\left|\Gamma_{l 2}\right|+|\gamma| \geqslant \frac{\epsilon}{2} .
\end{gathered}
$$

\subsection{Case II}

In Case II the reflection coefficient magnitudes $\left|\Gamma_{l 1}\right|$ and $\left|\Gamma_{a 1}\right|$ are given. In addition, it is assumed that $\Gamma_{a 2}=\Gamma_{l 2}$. As in the previous problem, the analysis is facilitated by considering a special case: $\Gamma_{l 1}=0$.

Subject to these conditions [eq (5-15)],

$$
\eta_{12}=\frac{|\alpha-\beta \gamma|}{1-|\gamma|^{2}}
$$

while

$$
\eta_{21}=\frac{|\alpha-\beta \gamma|\left(1-\left|\Gamma_{l 2}\right|^{2}\right)}{\left|1+\gamma \Gamma_{l 2}\right|^{2}-\left|\beta+\alpha \Gamma_{l 2}\right|^{2}}
$$

By hypothesis and by definition,

$$
\Gamma_{l 2}=\Gamma_{a 2}=-\gamma .
$$

10. Aside from the change in notation, the generalization of eqs (5-34)-(5-36) to (3-2) and (3-3) involves the recognition that $|\gamma|$ is identified with terminal surface 2 while the counterpart $\left|\Gamma_{a l}\right|$, as previously noted (footnote 3 ), is unspecified as to reference terminal. As a consequence, it is not possible to uniquely identify $\eta_{1}$ with either $\eta_{21}$ or $\eta_{12}$. Both alternatives must be considered in order to determine the largest possible error.

The "deterioration" in error limit in going from eqs $(5-34)-(5-36)$ to $(3-2)$ and $(3-3)$ is thus the "price" one pays for failure to identify the terminal surface associated with $\left|\Gamma_{n}\right|$. 
Moreover,

$$
\Gamma_{a 1}=\frac{\alpha \Gamma_{l 2}+\beta}{\gamma \Gamma_{l 2}+1}=\frac{\beta-\alpha \gamma}{1-\gamma^{2}} .
$$

By means of eqs (5-38) and (5-39) it is possible to eliminate $\beta$ and $\Gamma_{l 2}$ from eqs (5-15) and (5-37), resulting in

$$
\begin{aligned}
\eta_{21} & =\frac{\left|\alpha-\gamma \Gamma_{a 1}\right|\left(1-|\gamma|^{2}\right)}{\left|1-\gamma^{2}\right|\left(1-\left|\Gamma_{a 1}\right|^{2}\right)}, \\
\eta_{12} & =\frac{\left|\alpha-\gamma \Gamma_{a 1}\right|\left|1-\gamma^{2}\right|}{1-|\gamma|^{2}} .
\end{aligned}
$$

In a similar way eq $(5-8)$ becomes

$1-\frac{\left|\alpha-\gamma \Gamma_{a 1}\right|\left|1-\gamma^{2}\right|}{1-|\gamma|^{2}}$

$$
\geqslant \frac{\left|\alpha\left(\gamma-\gamma^{*}\right)+\Gamma_{a 1}\left(1-\gamma^{2}\right)\right|}{1-|\gamma|^{2}} \geqslant 0
$$

Let

$$
\frac{\alpha\left(\gamma-\gamma^{*}\right)+\Gamma_{a 1}\left(1-\gamma^{2}\right)}{1-|\gamma|^{2}}=\delta
$$

Equation (5-42) now becomes

$$
1-\eta_{12} \geqslant|\delta| \geqslant 0
$$

and eq (5-40) can be written:

$$
\eta_{21}=\frac{\eta_{12}^{2}-\left|\delta-\Gamma_{a 1}\right|^{2}}{\eta_{21}\left(1-\left|\Gamma_{a 1}\right|^{2}\right)}
$$

In general the value of $\delta$ will be unknown. However, it is evident that $\eta_{21}$ will have its maximum value if $\delta=\Gamma_{a 1}$. This in turn is possible provided $\left|\Gamma_{a 1}\right| \leqslant 1-\eta_{12}$, etc.

Therefore,

$$
\eta_{21(\max )}=\frac{\eta_{12}}{1-\left|\Gamma_{a 1}\right|^{2}} \quad \text { if }\left|\Gamma_{a 1}\right| \leqslant 1-\eta_{12},
$$

$$
\eta_{21(\max )}=\frac{2 \eta_{12}-\left(1-\left|\Gamma_{a 1}\right|\right)}{\eta_{12}\left(1+\left|\Gamma_{a 1}\right|\right)} \quad \text { if }\left|\Gamma_{a 1}\right| \geqslant 1-\eta_{12},
$$$$
\eta_{21(\min )}=\frac{2 \eta_{12}-\left(1+\left|\Gamma_{a 1}\right|\right)}{\eta_{12}\left(1-\left|\Gamma_{a 1}\right|\right)} .
$$

As in the previous problem, it is now possible to plot $\eta_{21(\max )}$ and $\eta_{21(\min )}$ in terms of $\eta_{12}$. It will then become apparent [as may also be shown by solving eqs $(5-46),(5-47)$, and $(5-48)$ for $\left.\eta_{12}\right]$ that this problem has been reduced to the previous one where the roles of $\eta_{12}$ and $\eta_{21}$ have been exchanged and $|\gamma|$ is replaced by $\left|\Gamma_{a 1}\right|$. The same arguments for extending the result to the case where $\Gamma_{l 1} \neq 0$ may also be used.

The expressions of interest may thus be obtained directly from eqs (5-34) and (5-35),

$$
\left.\frac{\eta_{21}}{\eta_{12}}\right|_{\max }=\left.\frac{\eta_{12}}{\eta_{21}}\right|_{\min } \text { etc. }
$$

Therefore, the approximate limits for Case II are

$$
\left(\frac{\eta_{21}}{\eta_{12}}\right)_{\max }^{1 / 2}=1+\frac{1}{2}\left(\left|\Gamma_{a 1}\right|+\left|\Gamma_{l 1}\right|\right)^{2}
$$

$$
\text { if }\left|\Gamma_{a 1}\right|+\left|\Gamma_{l 1}\right| \leqslant \frac{\epsilon}{2},
$$

$\left(\frac{\eta_{21}}{\eta_{12}}\right)_{\max }^{1 / 2}=1+\frac{\epsilon}{2}\left(\left|\Gamma_{a 1}\right|+\left|\Gamma_{l 1}\right|\right)-\frac{\epsilon^{2}}{8}$

$$
\text { if }\left|\Gamma_{a 1}\right|+\left|\Gamma_{l 1}\right| \geqslant \frac{\epsilon}{2},
$$

$\left(\frac{\eta_{21}}{\eta_{12}}\right)_{\min }^{1 / 2}=1-\frac{\epsilon}{2}\left(\left|\Gamma_{a 1}\right|+\left|\Gamma_{l 1}\right|\right)-\frac{\epsilon^{2}}{8}$.

\subsection{Case III}

The boundary conditions on Case III are $\Gamma_{l 1}=\Gamma_{a 1}$ (= $\Gamma$ for brevity) and $|\Gamma|$ is given. The treatment of the previous problems has been simplified by first assuming one or both loads to be matched, but this approach does not lend itself to the present problem. The analysis of Case III proceeds in a different manner.

The efficiencies may be written:

$$
\begin{aligned}
& \eta_{21}=\frac{|\alpha-\gamma \Gamma|^{2}-|\beta-\Gamma|^{2}}{|\alpha-\beta \gamma|\left(1-|\Gamma|^{2}\right)}, \\
& \eta_{12}=\frac{|\alpha-\beta \gamma|\left(1-|\Gamma|^{2}\right)}{|1-\beta \Gamma|^{2}-|\gamma-\alpha \Gamma|^{2}} .
\end{aligned}
$$

In terms of the previously defined parameters $x, y$ [eqs (5-9), (5-10)] these become

$$
\begin{gathered}
\eta_{21}=\frac{(1-x)^{2}-g^{2}}{(1-x)\left(1-|\Gamma|^{2}\right)}, \\
\eta_{12}=\frac{(1-x)\left(1-|\Gamma|^{2}\right)}{h^{2}-(1-x)^{2}|\Gamma|^{2}},
\end{gathered}
$$


where

$$
\begin{aligned}
& g=|y-\Gamma|, \\
& h=|1-y \Gamma| .
\end{aligned}
$$

Moreover

$$
\eta_{21} \eta_{12}=\frac{(1-x)^{2}-g^{2}}{h^{2}-(1-x)^{2}|\Gamma|^{2}}=\frac{1}{1+\epsilon}
$$

These equations may be combined to yield the ratio $\eta_{21} / \eta_{12}$ as a function of $\epsilon, \Gamma$, and $y .{ }^{11}$

$$
\frac{\eta_{21}}{\eta_{12}}=\frac{1+\epsilon}{\left(1+\epsilon+|\Gamma|^{2}\right)\left(1-|\Gamma|^{2}\right)^{2}} \cdot \frac{h^{2}\left(1-\frac{g^{2}}{h^{2}}|\Gamma|^{2}\right)^{2}}{1+\frac{g^{2}}{h^{2}}(1+\epsilon)}
$$

The terms $\epsilon$ and $|\Gamma|$ are parameters of the problem while $y$ is unknown and subject only to the restriction [eq $(5-11)]$

$$
|y| \leqslant x
$$

The problem is thus one of determining $y$ and $\arg \Gamma$ such that $\eta_{21} / \eta_{12}$ has its maximum and minimum values.

Inspection of eq (5-59) shows that $\eta_{21} / \eta_{12}$ decreases as $h$ decreases and as $g$ increases. Thus, the minimum value of $\eta_{21} / \eta_{12}$ occurs when $g=x+|\Gamma|$ and $h=1-x|\Gamma|$.

If these relations are now substituted into eq (5-58), the resulting equation may be solved for $x$ in terms of $\epsilon$ and $\Gamma$. This in turn determines $g$ and $h$ as functions of $\epsilon$ and I. Finally, substitution into eq (5-59) yields

$$
\begin{aligned}
\left.\frac{\eta_{21}}{\eta_{12}}\right|_{\min }=1-\frac{2 \epsilon|\Gamma|}{\left(1+\frac{\epsilon}{2}\right)\left(1+|\Gamma|^{2}\right)+\epsilon|\Gamma|} & \\
& -\frac{\frac{\epsilon^{2}}{4}\left(1-|\Gamma|^{2}\right)^{2}}{\left[\left(1+\frac{\epsilon}{2}\right)\left(1+|\Gamma|^{2}\right)+\epsilon|\Gamma|\right] 2},
\end{aligned}
$$

or if only the lowest order terms are retained,

$$
\left(\frac{\eta_{21}}{\eta_{12}}\right)_{\min }^{1 / 2}=1-\epsilon|\Gamma|-\frac{\epsilon^{2}}{8}
$$

Conversely, it is also evident from inspection that $\eta_{21} / \eta_{12}$ increases as $h$ increases and as $g$ decreases.

\footnotetext{
${ }^{11}$ Note that $g$ and $h$ are merely abbreviations for certain functions of $y$ and $\Gamma$.
}

Thus, the arguments of $y$ and $\Gamma$ should be chosen such that

$$
g=|| y|-| \Gamma||
$$

and

$$
h=1+|y \Gamma| .
$$

In this case, however, $|y|$ is not determined since $g_{\min }$ occurs for $|y|=|\Gamma|$ while $h_{\max }$ obtains for $|y|=x$. It thus becomes necessary to substitute the above expressions for $g$ and $h$ into eq (5-59) and differentiate with respect to $|y|$. The derivative vanishes and a maximum occurs for

$$
|y|=\frac{|\Gamma|(2+\epsilon)}{1+\epsilon-|\Gamma|^{2}}
$$

Substitution back into eq (5-59) leads to

$$
\frac{\eta_{21}}{\eta_{12 \max }}=\frac{\left(1+|\Gamma|^{2}\right)^{2}}{\left(1-|\Gamma|^{2}\right)^{2}}
$$

This however is subject to the condition that

$$
|y|=\frac{|\Gamma|(2+\epsilon)}{1+\epsilon-|\Gamma|^{2}} \leqslant x .
$$

The associated value of $x$ may now be computed by substituting eqs (5-62), (5-63), and (5-64) back into eq $(5-58)$. This leads to the condition that the solution given by equation (5-65) is valid in the range

$$
\frac{|\Gamma|(2+\epsilon)}{1+\epsilon-|\Gamma|^{2}} \leqslant 1-\frac{\left(1+|\Gamma|^{2}\right)(1+\epsilon)^{1 / 2}}{\left(1+\epsilon-|\Gamma|^{2}\right)},
$$

which may be solved to yield

$$
|\Gamma| \leqslant \frac{1}{\epsilon}\left[2+\epsilon-2(1+\epsilon)^{1 / 2}\right]=\frac{\epsilon}{4}-\frac{\epsilon^{2}}{8} . . .(5-68)
$$

For larger values of $|\Gamma|,|y|$ is set equal to $x$ and the problem handled as was done for $\eta_{21} /\left.\eta_{12}\right|_{\text {min }}$. Again, if only the lowest order terms are retained, $\left(\eta_{21} / \eta_{12}\right)_{\max }^{1 / 2}$ is given by

$$
\begin{array}{ll}
\left(\frac{\eta_{21}}{\eta_{12}}\right)_{\max }^{1 / 2}=1+2|\Gamma|^{2} & \text { if }|\Gamma| \leqslant \frac{\epsilon}{4}, \\
\left(\frac{\eta_{21}}{\eta_{12}}\right)_{\max }^{1 / 2}=1+\epsilon|\Gamma|-\frac{\epsilon^{2}}{8} & \text { if }|\Gamma| \geqslant \frac{\epsilon}{4} .
\end{array}
$$

With an appropriate change in notation, the results of this section leads to eqs $(3-2)-(3-9)$.

\section{Experimental Results}

An application of the foregoing techniques which is of immediate interest is the extension of the existing 
NBS calibration capability in X-band waveguide to coaxial thermistor and barretter mounts. A variety of these items, with an advertised upper frequency limit in the $10-12 \mathrm{GHz}$ range, is commercially available. A calibration near this upper limit is of particular interest because it appears reasonable to anticipate decreasing efficiency with increasing frequency. To the extent that this is true, the X-band calibration provides a lower limit to the efficiency over the entire operating range.

Measurements on a group of four mounts from different manufacturers (with Type $\mathrm{N}$ connectors) yielded efficiency values in the range 86-97 percent at $9 \mathrm{GHz}$. Another series of measurements on four different mounts but of the same make and model yielded values in the range 94-96.5 percent. These results indicate that high efficiency values are possible at X-band frequencies in coaxial mounts but also suggest there may be a much wider variation in different makes than is found in waveguide mounts.

The "waveguide-coax adaptor" used in the measurement was a commercial adaptor connected to a fivestub waveguide tuning transformer. The combination provided an average efficiency of approximately 98 percent.

Because of the Type $\mathrm{N}$ connectors, it was necessary to employ the procedures described in section 4 . The two measurements $m_{2 a}, m_{2 b}$ typically differed by 0.4 percent, thus the error limit from this source was \pm 0.1 percent. The error introduced by assuming $\sqrt{\eta_{21} / \eta_{12}}=1$ was computed from eqs $(3-7)-(3-9)$ and did not exceed \pm 0.1 percent. (The waveguide standard was matched with $\left|\Gamma_{w}\right|<0.01$.) These errors are in addition to those introduced by the calibration transfer procedure itself. Although this latter error can be held to a few tenths of a percent or so when the transfer is between waveguide mounts, the performance of certain of the coaxial components (sliding short, connector repeatability, etc.) is not up to that of the waveguide counterparts, and this calls for a wider estimate of the error limits. A tabulation of the errors in a typical calibration is thus as follows:

1. Uncertainty in efficiency of waveguide standard ................................

2. Calibration transfer procedure ........... $0.6-1.0 \%$

3. $\sqrt{\eta_{21} / 12}=1$ approximation................. $0.1 \%$

4. Difference in $m_{2 a}$ and $m_{2 b} \ldots \ldots \ldots \ldots \ldots . . . . . .0 .1 \%$

Total................................ $1.0-1.4 \%$

\section{Other Measurements}

Although attention has been focused primarily upon a specific application, the developed techniques have potential use in many other measurement problems where a change in waveguide is involved.

For example, the measurement of the adaptor efficiency may be the prime objective. Equation (2-4) may be combined with (3-1) to yield

$$
\eta_{1}=\sqrt{\eta_{1} \eta_{2}}(1+E)=\sqrt{\frac{m_{1}}{m_{2}}}(1+E) .
$$

The square root of the quotient of the two measurements thus yields the efficiency $\eta_{1}$ within the limits given for $E$. A similar expression may be obtained for $\eta_{2}$.

The author expresses his appreciation to several of his colleagues who provided experimental demonstrations of the technique, checked the mathematics, and provided constructive suggestions in the preparation of the manuscript. These include Mrs. Anne Rumfelt, Fred R. Clague, John W. Adams, and Robert W. Beatty.

(Paper 70C2-223) 